\title{
Some Miscellaneous Properties of Valuated Binary Tree
}

\author{
Xingbo WANG ${ }^{1,2}$, Shuai Zheng ${ }^{1,2}$, Li MA $^{2}$ \\ ${ }^{1}$ Department of Mechatronic Engineering, Foshan University, China \\ ${ }^{2}$ Department of Electronic Information,Foshan University, China \\ Correspondence: Xingbo WANG, Foshan University, Foshan City, 528000, China
}

Received: February 21, 2021 Accepted: March 30, 2021 Online Published: April 14, 2021

doi:10.5539/jmr.v13n3p1 URL: https://doi.org/10.5539/jmr.v13n3p1

\begin{abstract}
The paper proves several new properties of the valuated binary tree, including calculation of direct ancestors of a node, transition of a node in or out of a subtree, evaluation of a node by its brothers and distribution of common ancestors of given consecutive nodes. The contents can be a reference for future deep study of the valuated binary tree.
\end{abstract}

Keywords: valuated binary tree, odd integer, Node, ancestor, random walk

\section{Introduction}

The valuated binary tree has revealed many new outputs related with knowing of integers ever since it was introduced in paper WANG X (2016a) in 2016. For example, paper WANG X (2016b) showed the so-called "amusing properties of odd numbers", paper WANG X (2017a) disclosed some more symmetric properties of odd integers on the tree, paper WANG X (2017b) discovered genetic traits of the odd numbers on the tree, paper WANG X (2018a) investigated multiplication laws on $T_{3}$ tree, paper WANG X (2018b) made a research on square and square root of a node on the $T_{3}$ tree, paper WANG X (2019a) found some divisibility traits on the tree, papers WANG X (2019b) and WANG X (2019c) exhibited how the divisors of an RSA number distribute on the $T_{3}$ tree, and paper LI J (2018), which was based on the study of paper WANG X (2018c), proposed a parallel approach to factorize semiprimes. Over these researches, it can be seen that, the tree approach is demonstrating its capability in studying integers.

This paper, as a following research of the previous ones, shows some miscellaneous properties of the valuated tree. It aims at providing a mathematical foundation for some small but important mathematical reasoning for possible future studies on the tree.

\section{Preliminaries}

\subsection{Definitions $\mathcal{E}$ Notations}

A valuated binary tree $T$ is such a binary tree each of whose nodes is assigned a value. The terms binary tree and its root, nodes, father, left-son, right-son as well as subtrees can be seen in school-books of data structure, for example, Dinesh's handbook Dinesh $\mathrm{P}(2005)$. A positive odd integer $N$-rooted tree, denoted by $T_{N}$ is a recursively constructed valuated binary tree whose root is the odd integer $N$ with $2 N-1$ and $2 N+1$ being the root's left and right sons, respectively. Each son is connected with its father with a path, but there is no path between the two sons. The father, grandfather and so forth are called direct ancestors. Nodes on the same level are brothers. $T_{3}$ tree is the case $N=3$.

For convenience, symbol $N_{(k, j)}$ is by default the node at position $j$ on level $k$ of $T_{3}$, where $k \geq 0$ and $0 \leq j \leq 2^{k}-1$. Symbol $N_{(k, j)}^{N}$ is to denote the node at position $j$ on level $k$ of $T_{N}$, where $k \geq 0$ and $0 \leq j \leq 2^{k}-1$. When the index $j$ is out of the range $0 \leq j \leq 2^{k}-1$, for example, $j=-2,-1$ or $j=2^{k}, 2^{k}+1, N_{(k, j)}^{N}$ is called a virtual node or outer-node of $T_{N}$. $A_{N}^{\alpha}$ is $N$ 's direct ancestor that is $\alpha$ levels over $N$. Symbol $X \in l\left(T_{N}\right)$ means node $X$ is in the left branch of $T_{N}$ while symbol $X \in r\left(T_{N}\right)$ means node $X$ is in the right branch of $T_{N}$. An odd integer $N$ is said to align with level $k$ of a tree $T_{X}$ if $N$ and $N_{(k, *)}^{X}$ are on the same level of $T_{3}$. A walk of a node $N_{(k, j)}^{N}$ means an operation on the index $k$ or $j$, for example, $N_{(k+\sigma, j)}^{N}, N_{(k, j+\omega)}^{N}$ and $N_{(k+\sigma, j+\omega)}^{N}$ are all results from the walk of $N_{(k, j)}^{N}$. If either $\sigma$ or $\omega$ is taken randomly, the walk is called a random walk.

Symbol $A \Rightarrow B$ means result $B$ is derived from condition $A$ or $A$ can derive $B$ out. Symbol $A \otimes B\lfloor x\rfloor$ denotes the floor function, an integer function of the real number $x$ such that $x-1<\lfloor x\rfloor \leq x$ or equivalently $\lfloor x\rfloor \leq x<\lfloor x\rfloor+1$. Symbol $\{x\}=x-\lfloor x\rfloor$ is the fractional part of $x$. Symbol $f_{X}^{\alpha}$ is to express $\left\lfloor\frac{X-1}{2^{\alpha}}\right\rfloor$. 


\subsection{Lemmas}

Lemma 1(Node Calculation, see in WANG X (2016a, 2018a)). Odd integer $X>1$ lies on level $\left\lfloor\log _{2} X\right\rfloor-1$ of $T_{3}$ tree. Node $N_{(k, j)}$ of $T_{3}$ is calculated by

$$
\begin{aligned}
& N_{(k, j)}=2^{k+1}+1+2 j \\
& k=0,1,2, \ldots ; j=0,1, \ldots, 2^{k}-1
\end{aligned}
$$

Node $N_{(k, j)}^{X}$ of $T_{X}$ lies on level $\left\lfloor\log _{2} X\right\rfloor-1+k$ of $T_{X}$ and it is computed by

$$
\begin{aligned}
& N_{(k, j)}^{X}=2^{k} X-2^{k}+2 j+1 \\
& k=0,1,2, \ldots ; j=0,1, \ldots, 2^{k}-1
\end{aligned}
$$

Lemma 2(see in WANG X (2020a)). Properties of the floor functions with real numbers $x$ and $y$, integers $m, n$ and $k$

(P1) $\lfloor x\rfloor+\lfloor y\rfloor \leq\lfloor x+y\rfloor \leq\lfloor x\rfloor+\lfloor y\rfloor+1$

(P2) $\lfloor x\rfloor-\lfloor y\rfloor-1 \leq\lfloor x-y\rfloor \leq\lfloor x\rfloor-\lfloor y\rfloor<\lfloor x\rfloor-\lfloor y\rfloor+1$

(P13) $x \leq y \Rightarrow\lfloor x\rfloor \leq\lfloor y\rfloor$

(P14) $\lfloor x \pm n\rfloor=\lfloor x\rfloor \pm n$

(P32) $\lfloor n x\rfloor+1-n \leq n\lfloor x\rfloor \leq\lfloor n x\rfloor$

(P37) For an arbitrary positive integer $k$ and an arbitrary odd integer $N \geq 1$, the following holds

$$
\left\lfloor\frac{N}{2^{k}}\right\rfloor=\left\lfloor\frac{N-1}{2^{k}}\right\rfloor .
$$

Lemma 3. Odd Interval $[a, b]$ contains $\frac{b-a}{2}+1$ odd integers.

Proof. Assume $[a, b]$ contains $n$ odd integers; then $b=a+2(n-1)$ which leads to $\frac{b-a}{2}+1$.

\section{Main Results}

The following theorems, corollaries, propositions and inferences are newly found and proved. It is mandatory to point out first that, Lemma 2 is implicitly referred to in the whole reasoning process. Readers should keep this in mind when reading the proofs.

Theorem 1. Let $N>1$ be an odd integer on a tree; then $N$ 's direct ancestor that is $\alpha$ levels away from $N$ is calcualted by $A_{N}^{\alpha}=1+f_{N}^{\alpha}$ if $f_{N}^{\alpha}$ is even or $A_{N}^{\alpha}=f_{N}^{\alpha}$ if $f_{N}^{\alpha}$ is odd, where $f_{N}^{\alpha}=\left\lfloor\frac{N-1}{2^{\alpha}}\right\rfloor$.

Proof. Let $X$ be $N$ 's direct ancestor that is $\alpha$ levels away from $N$; then $N$ is on level $\alpha$ of $T_{X}$. Thereby by Lemma 2 (P13)

$$
\begin{aligned}
& 2^{\alpha}(X-1)+1 \leq N \leq 2^{\alpha}(X-1)+2\left(2^{\alpha}-1\right)+1 \\
& \Rightarrow X-1 \leq \frac{N-1}{2^{\alpha}} \leq(X-1)+2\left(1-\frac{1}{2^{\alpha}}\right)=X+1-\frac{1}{2^{\alpha-1}} \\
& \Rightarrow X-1 \leq\left\lfloor\frac{N-1}{2^{\alpha}}\right\rfloor \leq X .
\end{aligned}
$$

Since $X$ is odd, it is sure that the theorem holds.

Corollary 1. Let $N>1$ be an odd integer, $\alpha$ and $\beta$ be integers satisfying $1 \leq \alpha \leq \beta$; denoted $A_{l}^{\alpha}=A_{N+2}^{\alpha}$ and $A_{r}^{\alpha}=A_{N+2^{\beta}}^{\alpha}$; then the following holds

$$
A_{l}^{\alpha} \leq A_{r}^{\alpha} \leq A_{l}^{\alpha}+2^{\beta-\alpha}, 1 \leq \alpha<\beta
$$

and

$$
A_{l}^{\alpha} \leq A_{r}^{\alpha} \leq A_{l}^{\alpha}+2, \alpha=\beta
$$

Proof. Denote $F_{l}^{\alpha}=\left\lfloor\frac{N+2}{2^{\alpha}}\right\rfloor$ and $F_{r}^{\alpha}=\left\lfloor\frac{N+2^{\beta}}{2^{\alpha}}\right\rfloor$. For the case $1<\alpha<\beta$, it yields 


$$
\begin{aligned}
& F_{r}^{\alpha}=\left\lfloor\frac{N+2^{\beta}+2-2}{2^{\alpha}}\right\rfloor=\left\lfloor\frac{N+2}{2^{\alpha}}+2^{\beta-\alpha}-\frac{1}{2^{\alpha-1}}\right\rfloor \\
& \Rightarrow\left\lfloor\frac{N+2}{2^{\alpha}}\right\rfloor+2^{\beta-\alpha}+\left\lfloor-\frac{1}{2^{\alpha-1}}\right\rfloor \leq F_{r}^{\alpha} \leq\left\lfloor\frac{N+2}{2^{\alpha}}\right\rfloor+2^{\beta-\alpha}+\left\lfloor-\frac{1}{2^{\alpha-1}}\right\rfloor+1 \\
& \Rightarrow\left\lfloor\frac{N+2}{2^{\alpha}}\right\rfloor+2^{\beta-\alpha}-1 \leq F_{r}^{\alpha} \leq\left\lfloor\frac{N+2}{2^{\alpha}}\right\rfloor+2^{\beta-\alpha}
\end{aligned}
$$

which is

$$
F_{l}^{\alpha}+2^{\beta-\alpha}-1 \leq F_{r}^{\alpha} \leq F_{l}^{\alpha}+2^{\beta-\alpha}
$$

For the case $\alpha=1$, it yields

$$
\begin{aligned}
& F_{r}^{1}=\left\lfloor\frac{N+2^{\beta}+2-2}{2}\right\rfloor=\left\lfloor\frac{N+2}{2}+2^{\beta-1}-1\right\rfloor \\
& \Rightarrow F_{r}^{1}=\left\lfloor\frac{N+2}{2}\right\rfloor+2^{\beta-1}-1 .
\end{aligned}
$$

For the case $\alpha=\beta$, it yields

$$
\begin{aligned}
& F_{r}^{\beta}=\left\lfloor\frac{N+2^{\beta}+2-2}{2^{\beta}}\right\rfloor=\left\lfloor\frac{N+2}{2^{\beta}}+1-\frac{1}{2^{\beta-1}}\right\rfloor \\
& \Rightarrow\left\lfloor\frac{N+2}{2^{\beta}}\right\rfloor+1+\left\lfloor-\frac{1}{2^{\beta-1}}\right\rfloor \leq F_{r}^{\beta} \leq\left\lfloor\frac{N+2}{2^{\beta}}\right\rfloor+\left\lfloor-\frac{1}{2^{\beta-1}}\right\rfloor+2 \\
& \Rightarrow\left\lfloor\frac{N+2}{2^{\beta}}\right\rfloor \leq F_{r}^{\beta} \leq\left\lfloor\frac{N+2}{2^{\beta}}\right\rfloor+1 .
\end{aligned}
$$

Accordingly, by Theorem 1 the relationships among $F_{l}^{\alpha}, F_{r}^{\alpha}, A_{l}^{\alpha}$ and $A_{r}^{\alpha}$ are summarized in Table 1, which shows the corollary is true.

Table 1. Relationships among $F_{l}^{\alpha}, F_{r}^{\alpha}, A_{l}^{\alpha}$ and $A_{r}^{\alpha}$

\begin{tabular}{cll}
\hline$F_{l}^{\alpha}$ & $A_{l}^{\alpha}$ & $A_{r}^{\alpha}$ \\
\hline & & $F_{r}^{\alpha}=F_{l}^{\alpha}+2^{\beta-\alpha}-1 \Rightarrow A_{r}^{\alpha}=A_{l}^{\alpha}+2^{\beta-\alpha}, 1 \leq \alpha<\beta$ \\
$F_{l}^{\alpha}$ is odd & $A_{l}^{\alpha}=F_{l}^{\alpha}$ & $F_{r}^{\alpha}=F_{l}^{\alpha}+2^{\beta-\alpha}-1 \Rightarrow A_{r}^{\alpha}=A_{l}^{\alpha}, \alpha=\beta$ \\
& & $F_{r}^{\alpha}=F_{l}^{\alpha}+2^{\beta-\alpha} \Rightarrow A_{r}^{\alpha}=A_{l}^{\alpha}+2^{\beta-\alpha}, 1<\alpha<\beta$ \\
& $F_{r}^{\alpha}=F_{l}^{\alpha}+2^{\beta-\alpha} \Rightarrow A_{r}^{\alpha}=A_{l}^{\alpha}+2, \alpha=\beta$ \\
& & \\
& & $F_{r}^{\alpha}=F_{l}^{\alpha}+2^{\beta-\alpha}-1 \Rightarrow A_{r}^{\alpha}=A_{l}^{\alpha}+2^{\beta-\alpha}-2,1 \leq \alpha<\beta$ \\
& & $F_{r}^{\alpha}=F_{l}^{\alpha}+2^{\beta-\alpha}-1 \Rightarrow A_{r}^{\alpha}=A_{l}^{\alpha}, \alpha=\beta$ \\
$F_{l}^{\alpha}$ is even $\quad A_{l}^{\alpha}=F_{l}^{\alpha}+1 \quad F_{r}^{\alpha}=F_{l}^{\alpha}+2^{\beta-\alpha} \Rightarrow A_{r}^{\alpha}=A_{l}^{\alpha}+2^{\beta-\alpha}, 1<\alpha<\beta$ \\
& & $F_{r}^{\alpha}=F_{l}^{\alpha}+2^{\beta-\alpha} \Rightarrow A_{r}^{\alpha}=A_{l}^{\alpha}, \alpha=\beta$ \\
\hline
\end{tabular}

Corollary 2. Let $N>1$ be an odd integer, $\alpha$ and $\beta$ be integers satisfying $1 \leq \alpha \leq \beta$; denoted $A_{l}^{\alpha}=A_{N-2^{\beta}}^{\alpha}$ and $A_{r}^{\alpha}=A_{N-2}^{\alpha}$; then it holds

$$
A_{r}^{\alpha}-2^{\beta-\alpha} \leq A_{l}^{\alpha} \leq A_{r}^{\alpha}, 1 \leq \alpha<\beta
$$

and

$$
A_{r}^{\alpha}-2 \leq A_{l}^{\alpha} \leq A_{r}^{\alpha}, \alpha=\beta .
$$

Proof. Denote $F_{r}^{\alpha}=\left\lfloor\frac{N-2}{2^{\alpha}}\right\rfloor$ and $F_{l}^{\alpha}=\left\lfloor\frac{N-2^{\beta}}{2^{\alpha}}\right\rfloor$. For the case $1<\alpha<\beta$, it yields

$$
\begin{aligned}
& F_{l}^{\alpha}=\left\lfloor\frac{N-2^{\beta}+2-2}{2^{\alpha}}\right\rfloor=\left\lfloor\frac{N-2}{2^{\alpha}}-2^{\beta-\alpha}+\frac{1}{2^{\alpha-1}}\right\rfloor \\
& \Rightarrow\left\lfloor\frac{N-2}{2^{\alpha}}\right\rfloor-2^{\beta-\alpha}+\left\lfloor\frac{1}{2^{\alpha-1}}\right\rfloor \leq F_{l}^{\alpha} \leq\left\lfloor\frac{N-2}{2^{\alpha}}\right\rfloor-2^{\beta-\alpha}+\left\lfloor\frac{1}{2^{\alpha-1}}\right\rfloor+1 \\
& \Rightarrow\left\lfloor\frac{N+2}{2^{\alpha}}\right\rfloor-2^{\beta-\alpha} \leq F_{r}^{\alpha} \leq\left\lfloor\frac{N+2}{2^{\alpha}}\right\rfloor-2^{\beta-\alpha}+1
\end{aligned}
$$

which is

$$
F_{r}^{\alpha}-2^{\beta-\alpha} \leq F_{l}^{\alpha} \leq F_{r}^{\alpha}-2^{\beta-\alpha}+1
$$


For the case $\alpha=1$, it yields

$$
\begin{aligned}
& F_{l}^{1}=\left\lfloor\frac{N-2^{\beta}+2-2}{2}\right\rfloor=\left\lfloor\frac{N-2}{2}-2^{\beta-1}+1\right\rfloor \\
& \Rightarrow F_{l}^{1}=\left\lfloor\frac{N-2}{2}\right\rfloor-2^{\beta-1}+1 .
\end{aligned}
$$

For the case $\alpha=\beta$, it yields

$$
\begin{aligned}
& F_{l}^{\alpha}=\left\lfloor\frac{N-2^{\beta}+2-2}{2^{\beta}}\right\rfloor=\left\lfloor\frac{N-2}{2^{\beta}}-1+\frac{1}{2^{\beta-1}}\right\rfloor \\
& \Rightarrow\left\lfloor\frac{N-2}{2^{\beta}}\right\rfloor-1+\left\lfloor\frac{1}{2^{2-1}}\right\rfloor \leq F_{l}^{\alpha} \leq\left\lfloor\frac{N-2}{2^{\alpha}}\right\rfloor-1+\left\lfloor\frac{1}{2^{\alpha-1}}\right\rfloor+1 \\
& \Rightarrow\left\lfloor\frac{N+2}{2^{\alpha}}\right\rfloor-2^{\beta-\alpha}-1 \leq F_{r}^{\alpha} \leq\left\lfloor\frac{N+2}{2^{\alpha}}\right\rfloor-2^{\beta-\alpha} .
\end{aligned}
$$

The relationships among $F_{l}^{\alpha}, F_{r}^{\alpha}, A_{l}^{\alpha}$ and $A_{r}^{\alpha}$ are summarized in Table 1, which shows the corollary is true.

Table 2. Relationships among $F_{l}^{\alpha}, F_{r}^{\alpha}, A_{l}^{\alpha}$ and $A_{r}^{\alpha}$

\begin{tabular}{cll}
\hline$F_{r}^{\alpha}$ & $A_{r}^{\alpha}$ & $A_{l}^{\alpha}$ \\
\hline & & $F_{l}^{\alpha}=F_{r}^{\alpha}-2^{\beta-\alpha} \Rightarrow A_{l}^{\alpha}=A_{r}^{\alpha}-2^{\beta-\alpha}, 1<\alpha<\beta$ \\
$F_{r}^{\alpha}$ is odd & $A_{r}^{\alpha}=F_{r}^{\alpha}$ & $F_{l}^{\alpha}=F_{r}^{\alpha}-2^{\beta-\alpha} \Rightarrow A_{l}^{\alpha}=A_{r}^{\alpha}, \alpha=\beta$ \\
& & $F_{l}^{\alpha}=F_{r}^{\alpha}-2^{\beta-\alpha}+1 \Rightarrow A_{l}^{\alpha}=A_{r}^{\alpha}-2^{\beta-\alpha}+2,1 \leq \alpha<\beta$ \\
& $F_{l}^{\alpha}=F_{r}^{\alpha}-2^{\beta-\alpha}+1 \Rightarrow A_{l}^{\alpha}=A_{r}^{\alpha}, \alpha=\beta$ \\
& & \\
& & $F_{l}^{\alpha}=F_{r}^{\alpha}-2^{\beta-\alpha} \Rightarrow A_{l}^{\alpha}=A_{r}^{\alpha}-2^{\beta-\alpha}, 1<\alpha<\beta$ \\
$F_{r}^{\alpha}$ is even & $A_{r}^{\alpha}=F_{r}^{\alpha}+1 \quad F_{l}^{\alpha}=F_{r}^{\alpha}-2^{\beta-\alpha} \Rightarrow A_{l}^{\alpha}=A_{r}^{\alpha}-2, \alpha=\beta$ \\
& & $F_{l}^{\alpha}=F_{r}^{\alpha}-2^{\beta-\alpha}+1 \Rightarrow A_{l}^{\alpha}=A_{r}^{\alpha}-2^{\beta-\alpha}, 1 \leq \alpha<\beta$ \\
& & $F_{l}^{\alpha}=F_{r}^{\alpha}-2^{\beta-\alpha}+1 \Rightarrow A_{l}^{\alpha}=A_{r}^{\alpha}, \alpha=\beta$
\end{tabular}

Proposition 1. Let $X=2^{\alpha} u+1$ or $X=2^{\alpha} u-1$ with $\alpha \geq 1$ being an integer and $u>1$ being an odd integer. Then

$$
X \in T_{u} \text {. }
$$

Proof. Direct calculations yield

$$
\begin{gathered}
X=2^{\alpha} u+1=2^{\alpha}(u-1)+2\left(2^{\alpha-1}\right)+1=N_{\left(\alpha, 2^{\alpha-1}\right)}^{u} \in T_{u} \\
X=2^{\alpha} u-1=2^{\alpha}(u-1)+2\left(2^{\alpha-1}-1\right)+1=N_{\left(\alpha, 2^{\alpha-1}-1\right)}^{u} \in T_{u} .
\end{gathered}
$$

Proposition 2. Let $X>1$ be an odd integer and $\alpha>0$ be an integer; assume $Y=N_{(\alpha, \omega)}^{X}$; then one of $Y+2^{\alpha}$ and $Y-2^{\alpha}$ lies on level $\alpha$ of $T_{X}$.

Proof. By Lemma 1

$$
\begin{aligned}
& Y+2^{\alpha}=N_{(\alpha, \omega)}^{X}+2^{\alpha}=2^{\alpha}(X-1)+2\left(\omega+2^{\alpha-1}\right)+1 \\
& Y-2^{\alpha}=N_{(\alpha, \omega)}^{X}+2^{\alpha}=2^{\alpha}(X-1)+2\left(\omega-2^{\alpha-1}\right)+1
\end{aligned}
$$

Obviously, the following reasoning processes are true

$$
\begin{aligned}
& Y \in l\left(T_{X}\right) \Rightarrow 0 \leq \omega \leq 2^{\alpha-1}-1 \\
& \Rightarrow 2^{\alpha-1} \leq \omega+2^{\alpha-1} \leq 2^{\alpha}-1 \\
& \Rightarrow Y+2^{\alpha} \in r\left(T_{X}\right)
\end{aligned}
$$

and

$$
\begin{aligned}
& Y \in r\left(T_{X}\right) \Rightarrow 2^{\alpha-1} \leq \omega \leq 2^{\alpha}-1 \\
& \Rightarrow 0 \leq \omega-2^{\alpha-1} \leq 2^{\alpha-1}-1 \\
& \Rightarrow Y-2^{\alpha} \in l\left(T_{X}\right) .
\end{aligned}
$$


Proposition 3. Let $X>1$ be an odd integer and $\alpha$ be an integer; then the following holds in $T_{X}$

$$
\begin{gathered}
N_{\left(\alpha, 2^{\alpha}-1\right)}^{X}-N_{(\alpha, 0)}^{X}=2\left(2^{\alpha}-1\right)=2^{\alpha+1}-2, \alpha \geq 0 \\
N_{\left(\alpha, 2^{\alpha-1}\right)}^{X}-N_{(\alpha, 0)}^{X}=2^{\alpha}, \alpha>0 \\
N_{\left(\alpha, 2^{\alpha}-1\right)}^{X}-N_{\left(\alpha, 2^{\alpha-1}-1\right)}^{X}=2^{\alpha}, \alpha>0 .
\end{gathered}
$$

Proof. By Lemma $1, N_{(\alpha, 0)}^{X}=2^{\alpha}(X-1)+1, N_{\left(\alpha, 2^{\alpha-1}\right)}^{X}=2^{\alpha}(X-1)+2\left(2^{\alpha-1}-1\right)+1, N_{\left(\alpha, 2^{\alpha-1}-1\right)}^{X}=2^{\alpha}(X-1)+2\left(2^{\alpha-1}\right)+1, N_{\left(\alpha, 2^{\alpha}-1\right)}^{X}=$ $2^{\alpha}(X-1)+2\left(2^{\alpha}-1\right)+1$. Hence $N_{\left(\alpha, 2^{\alpha}-1\right)}^{X}-N_{(\alpha, 0)}^{X}=2\left(2^{\alpha}-1\right), N_{\left(\alpha, 2^{\alpha-1}\right)}^{X}-N_{(\alpha, 0)}^{X}=2^{\alpha}$ and $N_{\left(\alpha, 2^{\alpha}-1\right)}^{X}-N_{\left(\alpha, 2^{\alpha-1}-1\right)}^{X}=2^{\alpha}$.

Inference 1. If positive odd integers $X$ and $N$ lie on level $\alpha$ of tree $T_{A}$ with integer $\alpha \geq 0$; then $|N-X| \leq 2\left(2^{\alpha}-1\right)$.

Proof. $X$ and $N$ lying on level $\alpha$ yields $|N-X| \leq N_{\left(\alpha, 2^{\alpha}-1\right)}^{A}-N_{(\alpha, 0)}^{A}=2\left(2^{\alpha}-1\right)$.

Remark 1. The converse of Inference 1 is not true. For example, 17 and 19 lie on different levels of $T_{3}$.

Proposition 4. Let $X>1$ be an odd integer and $\alpha>0$ be an integer. Then

$$
N_{(\alpha, \omega)}^{X+2 k}-N_{(\alpha, \omega)}^{X}=2^{\alpha+1} k
$$

and accordingly for an arbitrary integer $\beta>0$

$$
N_{(\alpha, \omega)}^{X+2^{\beta}}-N_{(\alpha, \omega)}^{X}=2^{\alpha+\beta}
$$

Proof. Directly calculation yields $N_{(\alpha, \omega)}^{X+2 k}-N_{(\alpha, \omega)}^{X}=2^{\alpha}(X+2 k-1)+2 \omega+1-2^{\alpha}(X-1)-2 \omega-1=2^{\alpha+1} k$.

Proposition 5. For a positive integer $\beta$, suppose $X>0$ is an odd integer and $Y=X+2^{\beta}$; then

$$
A_{Y}^{\beta-1}=A_{X}^{\beta-1}+2
$$

and

$$
A_{X}^{\beta} \leq A_{Y}^{\beta} \leq A_{X}^{\beta}+2
$$

Proof. Direct calculating follows

$$
\begin{aligned}
& \frac{Y-1}{2^{\beta-1}}=\frac{X-1}{2^{\beta-1}}+2 \\
& \Rightarrow\left\lfloor\frac{Y-1}{2^{\beta-1}}\right\rfloor=\left\lfloor\frac{X-1}{2^{\beta-1}}\right\rfloor+2 \\
& \Rightarrow A_{Y}^{\beta-1}=A_{X}^{\beta-1}+2
\end{aligned}
$$

and

$$
\begin{aligned}
& \frac{Y-1}{2^{\beta}}=\frac{X-1}{2^{\beta}}+1 \\
& \Rightarrow\left\lfloor\frac{Y-1}{2^{\beta}}\right]^{=}=\left\lfloor\frac{X-1}{2^{\beta}}\right\rfloor+1 .
\end{aligned}
$$

Let $f_{Y}^{\beta}=\left\lfloor\frac{Y-1}{2^{\beta}}\right\rfloor$ and $f_{X}^{\beta}=\left\lfloor\frac{X-1}{2^{\beta}}\right\rfloor$; then relationships among $f_{X}^{\beta}, f_{Y}^{\beta}, A_{X}^{\beta}$ and $A_{Y}^{\beta}$ are summarized in Table 3 , which shows the proposition is true.

Table 3. Relationships among $f_{X}^{\beta}, f_{Y}^{\beta}, A_{X}^{\beta}$ and $A_{Y}^{\beta}$

\begin{tabular}{ccl}
\hline$f_{X}^{\beta}$ & $A_{X}^{\beta}$ & $A_{Y}^{\beta}$ \\
\hline$f_{X}^{\beta}$ is odd & $A_{X}^{\beta}=f_{X}^{\beta}$ & $A_{Y}^{\beta}=f_{Y}^{\beta}+1 \Rightarrow A_{Y}^{\beta}=f_{X}^{\beta}+2=A_{X}^{\beta}+2$ \\
$f_{X}^{\beta}$ ie even & $A_{X}^{\beta}=f_{X}^{\beta}+1$ & $A_{Y}^{\beta}=f_{Y}^{\beta} \Rightarrow A_{Y}^{\beta}=f_{X}^{\beta}+1=A_{X}^{\beta}$ \\
\hline
\end{tabular}

Proposition 6. For a positive integer $\beta$, suppose $X>0$ is an odd integer and $Y=X-2^{\beta}$; then

$$
A_{Y}^{\beta-1}=A_{X}^{\beta-1}-2
$$

and

$$
A_{X}^{\beta}-2 \leq A_{Y}^{\beta} \leq A_{X}^{\beta}
$$


Proof. Direct calculating follows

$$
\begin{aligned}
& \frac{Y-1}{2^{\beta-1}}=\frac{X-1}{2^{\beta-1}}-2 \\
& \Rightarrow\left\lfloor\frac{Y-1}{2^{\beta-1}}\right\rfloor=\left\lfloor\frac{X-1}{2^{\beta-1}}\right\rfloor-2 \\
& \Rightarrow A_{Y}^{\beta-1}=A_{X}^{\beta-1}-2
\end{aligned}
$$

and

$$
\begin{aligned}
& \frac{Y-1}{2^{\beta}}=\frac{X-1}{2^{\beta}}-1 \\
& \Rightarrow\left\lfloor\frac{Y-1}{2^{\beta}}\right]^{=}=\left[\frac{X-1}{2^{\beta}}\right\rfloor-1 .
\end{aligned}
$$

Let $f_{Y}^{\beta}=\left\lfloor\frac{Y-1}{2^{\beta}}\right\rfloor$ and $f_{X}^{\beta}=\left\lfloor\frac{X-1}{2^{\beta}}\right\rfloor$. Then referring to the proof of Proposition 5 results in the conclusions.

Remark 2. Propositions 5 and 6 show that, for an arbitrary positive integer $\beta, 2^{\beta-1}+1$ consecutive positive odd integers might lie in a subtree or in two adjacent subtrees of $T_{3}$. In another word, it always can find one subtree or two adjacent subtrees to have $2^{\beta-1}+1$ consecutive positive odd integers to be descendants.

Proposition 7. Let $N>1$ be an odd integer on a tree, $f_{N}^{\alpha}=\left\lfloor\frac{N-1}{2^{\alpha}}\right\rfloor$ and $A_{N}^{\alpha}$ be $N$ 's direct ancestor that is $\alpha$ levels away from $N$; then when $f_{N}^{\alpha}$ is even

$$
N \leq 2^{\alpha} A_{N}^{\alpha} \leq 2^{\alpha}+N-1
$$

whereas when $f_{N}^{\alpha}$ is odd

$$
N-2^{\alpha} \leq 2^{\alpha} A_{N}^{\alpha} \leq N-1 .
$$

Proof. First consider the case that $f_{N}^{\alpha}$ is even. This time $A_{N}^{\alpha}=1+f_{N}^{\alpha}$ and

$$
2^{\beta} A_{N}^{\alpha}=2^{\beta}+2^{\beta}\left\lfloor\frac{N-1}{2^{\alpha}}\right\rfloor .
$$

By Lemma 2(P32), it knows

$$
2^{\beta-\alpha}(N-1)+1 \leq 2^{\beta} A_{N}^{\alpha} \leq 2^{\beta}+2^{\beta-\alpha}(N-1) .
$$

When $f_{N}^{\alpha}$ is odd, $A_{N}^{\alpha}=f_{N}^{\alpha}$ and thus

$$
2^{\beta-\alpha}(N-1)-2^{\beta}+1 \leq 2^{\beta} A_{N}^{\alpha}=2^{\beta}\left\lfloor\frac{N-1}{2^{\alpha}}\right\rfloor \leq 2^{\beta-\alpha}(N-1) .
$$

Under the condition $\beta=\alpha$, it holds

$$
N \leq 2^{\alpha} A_{N}^{\alpha} \leq 2^{\alpha}+N-1
$$

or

$$
N-2^{\alpha} \leq 2^{\alpha} A_{N}^{\alpha} \leq N-1 .
$$

Proposition 8. Let $N>1$ be an odd integer on a tree $T, A_{N}^{\alpha}$ and $A_{N}^{\alpha+1}$ be $N$ 's two direct ancestors; denote $l_{N}^{\alpha+1}$ and $r_{N}^{\alpha+1}$ to be respectively the leftmost and rightmost nodes on $N$ 's level that are rooted with $A_{N}^{\alpha+1}, l_{N}^{\alpha}$ and $r_{N}^{\alpha}$ to be the leftmost and rightmost nodes respectively of $N$ 's level that are rooted with $A_{N}^{\alpha}$. Then

$$
A_{N}^{\alpha} \in l\left(A_{N}^{\alpha+1}\right) \Rightarrow r_{N}^{\alpha+1}-r_{N}^{\alpha}=2^{\alpha+1}, A_{N}^{\alpha} \in r\left(A_{N}^{\alpha+1}\right) \Rightarrow r_{N}^{\alpha+1}-r_{N}^{\alpha}=0
$$

and

$$
A_{N}^{\alpha} \in l\left(A_{N}^{\alpha+1}\right) \Rightarrow l_{N}^{\alpha+1}-r_{N}^{\alpha}=0, A_{N}^{\alpha} \in r\left(A_{N}^{\alpha+1}\right) \Rightarrow l_{N}^{\alpha+1}-r_{N}^{\alpha}=-2^{\alpha+1} .
$$

Proof. By Lemma 1 the following holds 


$$
\begin{aligned}
& \left(r_{N}^{\alpha+1}=2^{\alpha+1} A_{N}^{\alpha+1}+2^{\alpha+1}-1\right) \otimes\left(r_{N}^{\alpha}=2^{\alpha} A_{N}^{\alpha}+2^{\alpha}-1\right) \\
& \Rightarrow r_{N}^{\alpha+1}-r_{N}^{\alpha}=2^{\alpha+1} A_{N}^{\alpha+1}-2^{\alpha} A_{N}^{\alpha}+2^{\alpha} . \\
& \Rightarrow r_{N}^{\alpha+1}-r_{N}^{\alpha}=2^{\alpha}\left(2 A_{N}^{\alpha+1}-A_{N}^{\alpha}\right)+2^{\alpha} .
\end{aligned}
$$

Likewise

$$
l_{N}^{\alpha+1}-l_{N}^{\alpha}=2^{\alpha}\left(2 A_{N}^{\alpha+1}-A_{N}^{\alpha}\right)-2^{\alpha} .
$$

Since

$$
\begin{aligned}
& A_{N}^{\alpha}=\left\{\begin{array}{l}
2 A_{N}^{\alpha+1}-1, A_{N}^{\alpha} \in l\left(A_{N}^{\alpha+1}\right) \\
2 A_{N}^{\alpha+1}+1, A_{N}^{\alpha} \in r\left(A_{N}^{\alpha+1}\right)
\end{array}\right. \\
& \Rightarrow 2 A_{N}^{\alpha+1}=\left\{\begin{array}{l}
A_{N}^{\alpha}+1, A_{N}^{\alpha} \in l\left(A_{N}^{\alpha+1}\right) \\
A_{N}^{\alpha}-1, A_{N}^{\alpha} \in r\left(A_{N}^{\alpha+1}\right)
\end{array}\right.
\end{aligned}
$$

it yields

$$
r_{N}^{\alpha+1}-r_{N}^{\alpha}=\left\{\begin{array}{c}
0, A_{N}^{\alpha} \in r\left(A_{N}^{\alpha+1}\right) \\
2^{\alpha+1}, A_{N}^{\alpha} \in l\left(A_{N}^{\alpha+1}\right)
\end{array}\right.
$$

and

$$
l_{N}^{\alpha+1}-l_{N}^{\alpha}=\left\{\begin{array}{c}
-2^{\alpha+1}, A_{N}^{\alpha} \in r\left(A_{N}^{\alpha+1}\right) \\
0, A_{N}^{\alpha} \in l\left(A_{N}^{\alpha+1}\right)
\end{array} .\right.
$$

The proproty described in Proposition 8 can be described with figure 1 .

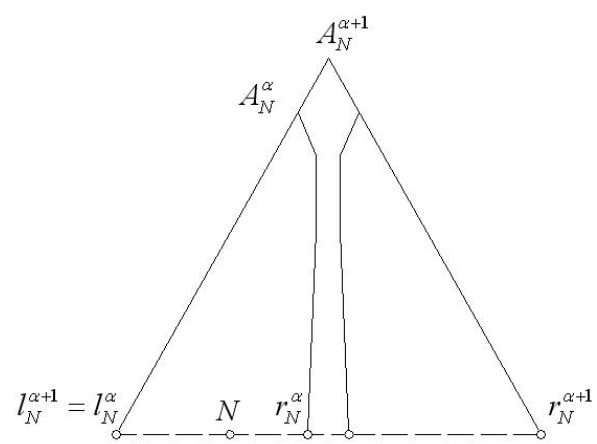

(a) $A_{N}^{\alpha} \in l\left(A_{N}^{\alpha+1}\right)$

Figure 1.Boundary property

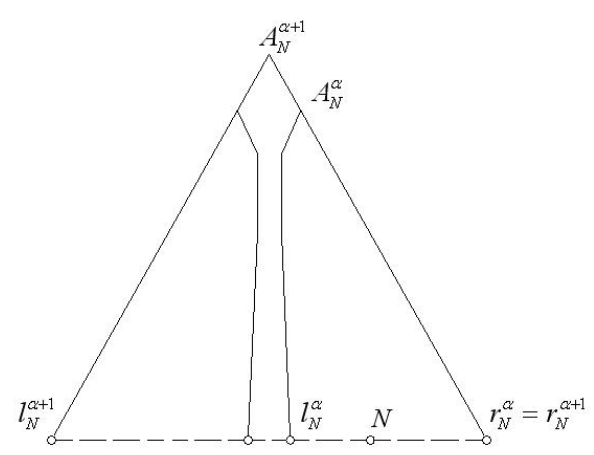

(b) $A_{N}^{\alpha} \in r\left(A_{N}^{\alpha+1}\right)$

Proposition 9. In $T_{X}$ tree it holds for integers $k>0$ and $0 \leq j \leq 2^{k}-1$

$$
\begin{gathered}
N_{\left(k+1,2^{k-1}+j\right)}^{X}=N_{(k, j)}^{X}+2^{k} X \\
N_{(k, j)}^{X}+2^{k} X-2^{k}=N_{(k+1, j)}^{X} \in T_{X}
\end{gathered}
$$

and

$$
N_{(k, j)}^{X}+2^{k} X+2^{k}=N_{\left(k+1,2^{k}+j\right)}^{X} \in T_{X}
$$

and accordingly

$$
N_{(k, j)}^{X}+2^{k} X+2 \omega \in T_{X}
$$

where $-2^{k-1} \leq \omega \leq 2^{k-1}$.

Proof. By Lemma 1, see the following calculations

$$
\begin{aligned}
& N_{(k, j)}^{X}=2^{k} X-2^{k}+2 j+1 \\
& \Rightarrow N_{(k, j)}^{X}+2^{k} X=2^{k+1} X-2^{k+1}+2^{k}+2 j+1=N_{\left(k+1,2^{k-1}+j\right)}^{X}
\end{aligned}
$$




$$
\begin{aligned}
& N_{(k, j)}^{X}=2^{k} X-2^{k}+2 j+1 \\
& \Rightarrow N_{(k, j)}^{X}+2^{k} X-2^{k}=2^{k+1} X-2^{k+1}+2 j+1=N_{(k+1, j)}^{X}
\end{aligned}
$$

and

$$
\begin{aligned}
& N_{\left(k+1,2^{k}+j\right)}^{X}-N_{(k, j)}^{X} \\
& =2^{k+1} X-2^{k+1}+2\left(2^{k}+j\right)+1-\left(2^{k} X-2^{k}+2 j+1\right) \\
& =2^{k} X+2^{k}
\end{aligned}
$$

Note that, when $-2^{k-1} \leq \omega \leq 2^{k-1}$, it holds

$$
N_{(k+1, j)}^{X} \leq N_{(k, j)}^{X}+2^{k} X+2 \omega \leq N_{\left(k+1,2^{k}+j\right)}^{X}
$$

and thus

$$
N_{(k, j)}^{X}+2^{k} X+2 \omega \in T_{X} .
$$

Proposition 10. For $T_{X}$ and integer $k \geq 0$, it holds

$$
N_{\left(k+1,2^{k}-1 \pm \omega\right)}^{X}=N_{\left(k, 2^{k-1}-1 \pm \omega\right)}^{X} \pm 2^{k} X
$$

and

$$
N_{\left(k+1,2^{k} \pm \omega\right)}^{X}=N_{\left(k, 2^{k-1} \pm \omega\right)}^{X} \pm 2^{k} X
$$

where $\omega$ is an integer satisfying $0 \leq \omega \leq 2^{\left\lfloor\log _{2} X\right\rfloor-1+k}$ and the \pm symbols are mandatory to be the same in the corresponding terms, namely, one term taking + requires the other terms to take + , or vice versa.

Proof. By Lemma $1, X$ lies on level $\left\lfloor\log _{2} X\right\rfloor-1$ of $T_{3}$ thus $N_{\left(k, 2^{k-1}-1+\omega\right)}^{X}$ lies on level $\left\lfloor\log _{2} X\right\rfloor+k-1$ of $T_{3}$. That level of

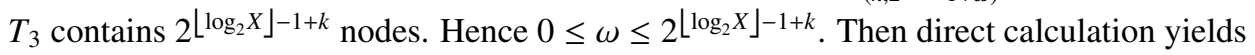

$$
\begin{aligned}
& N_{\left(k+1,2^{k}-1 \pm \omega\right)}^{X}-N_{\left(k, 2^{k-1}-1 \pm \omega\right)}^{X} \\
& =2^{k+1} X-2^{k+1}+2\left(2^{k}-1 \pm \omega\right)+1-\left(2^{k} X-2^{k}+2\left(2^{k-1}-1 \pm \omega\right)+1\right) \\
& =2^{k} X .
\end{aligned}
$$

Likewise, direct calculation yields

$$
N_{\left(k+1,2^{k} \pm \omega\right)}^{X}-N_{\left(k, 2^{k-1} \pm \omega\right)}^{X}=2^{k} X
$$

Proposition 11. Let $N=N_{(k, j)} \in T_{3}, \sigma \geq 0$ be an integer, $\omega$ and $\theta$ be integers satisfying $0 \leq \omega \leq 2^{k+\sigma}-1-j$ and $0 \leq \theta \leq j$; then

$$
N+2^{k+1}\left(2^{\sigma}-1\right)+2 \omega=N_{(k+\sigma, j+\omega)}
$$

and

$$
N+2^{k+1}\left(2^{\sigma}-1\right)-2 \theta=N_{(k+\sigma, j-\theta)} .
$$

Proof. First it can see

$$
\begin{aligned}
& N+2^{k+1}=N_{(k+1, j)} \\
& \Rightarrow N_{(k+1, j)}+2^{k+2}=N+2^{k+1}+2^{k+2}=N_{(k+2, j)} \\
& \cdots \\
& \Rightarrow\left(N+2^{k+1}+2^{k+2}+\ldots 2^{k+\sigma-1}\right)+2^{k+\sigma}=N_{(k+\sigma-1, j)}+2^{k+\sigma}=N_{(k+\sigma, j)} .
\end{aligned}
$$

That is

$$
N+2^{k+1}\left(2^{\sigma}-1\right)=N_{(k+\sigma, j)} .
$$

Note that, $0 \leq \omega \leq 2^{k+\sigma}-1-j \Rightarrow j \leq j+\omega \leq 2^{k+\sigma}-1, N_{(k+\sigma, j)}+2 \omega=N_{(k+\sigma, j+\omega)}$, and surely

$$
N+2^{k+1}\left(2^{\sigma}-1\right)+2 \omega=N_{(k+\sigma, j+\omega)}
$$


Likewise, $0 \leq \theta \leq j \Rightarrow 0 \leq j-\theta \leq j$ yields

$$
N_{(k+\sigma, j)}-2 \theta=N_{(k+\sigma, j-\theta)}
$$

and

$$
N+2^{k+1}\left(2^{\sigma}-1\right)-2 \theta=N_{(k+\sigma, j-\theta)} .
$$

Remark 3. The formulas in Proposition 11 reveal a property related with the walk of a node. One can think the node $N+2^{k+1}\left(2^{\sigma}-1\right)+2 \omega=N_{(k+\sigma, j+\omega)}$ comes from $N$ 's walking downward by $\sigma$ steps and then rightward by $\omega$ steps. This is why we prefer to this proof rather than another one that obtains $N+2^{k+1}\left(2^{\sigma}-1\right)+2 \omega=N_{(k+\sigma, j+\omega)}$ and $N+2^{k+1}\left(2^{\sigma}-1\right)-2 \theta=$ $N_{(k+\sigma, j-\theta)}$ directly by $N=2^{k+1}+2 j+1$.

Proposition 12. Given an positive odd integer $N$ and a positive integer $\beta$ satisfying $1 \leq \beta<\left\lfloor\log _{2} N\right\rfloor$; let $X$ be an odd integer in odd interval $I=\left[N+2, N+2^{\beta}\right]$. Then

$$
A_{N+2}^{\beta} \leq A_{X}^{\beta} \leq A_{N+2}^{\beta}+2 .
$$

Proof. The conclusion of this proposition is actually established in Corollary 2. However we'd like to present a detailed proof so as to make reader know the deep meaning of this proposition. The case $\beta=1$ yields $X=N+2$. Hence we next consider $1<\beta \leq\left\lfloor\log _{2} N\right\rfloor-1$. Since the interval contains $n_{\beta}=2^{\beta-1}$ nodes, the following two cases might occur.

Case 1.The whole interval lies on a same level of $T_{3}$. It can prove that, this time the $n_{\beta}$ nodes might be descendants of one ancestor $A$ or descendants of two ancestors, $A$ and $A+2$, as illustrated in figure 2.

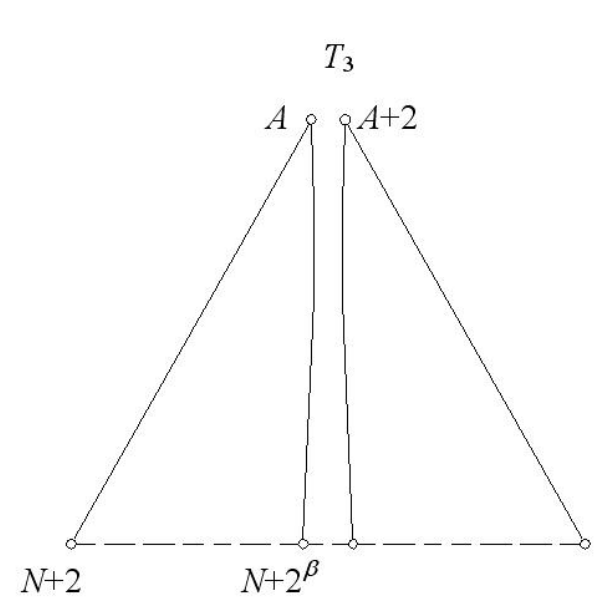

(a) $n_{\beta}$ nodes are descendants of $A$

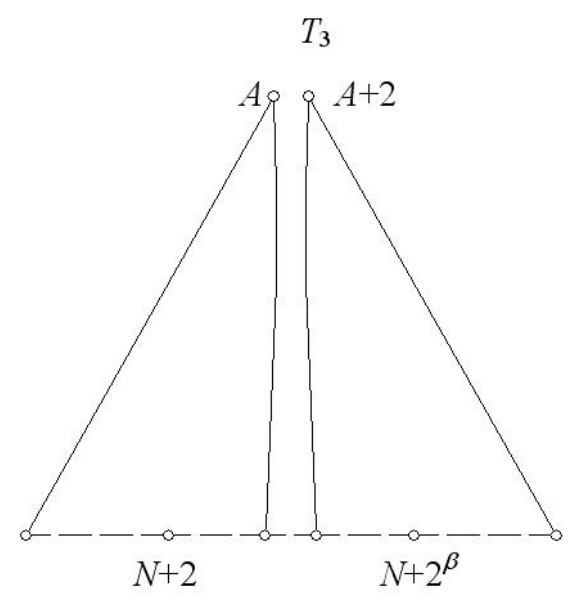

(b) $n_{\beta}$ nodes are descendants of $A$ and $A+2$

Figure 2. $2^{\beta-1}$ consecutive nodes lie on a level of $T_{3}$

Actually, consider the ancestors of $N+2$ and $N+2^{\beta}$ on the level that are $\beta$ levels upper $N+2$ or $N+2^{\beta}$. Let $A_{l}^{\beta}$ and $A_{r}^{\beta}$ be the ancestors of $N+2$ and $N+2^{\beta}$, respectively. Let $F_{l}^{\beta}=\left\lfloor\frac{N+2}{2^{\beta}}\right\rfloor$ and $F_{r}^{\beta}=\left\lfloor\frac{N+2^{\beta}}{2^{\beta}}\right\rfloor$; then by Theorem $1 A_{l}^{\beta}$ takes its value by the odd one between $F_{l}^{\beta}$ and $F_{l}^{\beta}+1$ and $A_{r}^{\beta}$ takes its value by the odd one between $F_{r}^{\beta}$ and $F_{r}^{\beta}+1$. Note that,

$$
\begin{gathered}
F_{r}^{\beta}=\left\lfloor\frac{N+2^{\beta}+2-2}{2^{\beta}}\right\rfloor=\left\lfloor\frac{N+2}{2^{\beta}}+1-\frac{1}{2^{\beta-1}}\right\rfloor \\
F_{r}^{\beta}=\left\lfloor\frac{N+2^{\beta}-1}{2^{\beta}}\right\rfloor=\left\lfloor\frac{(N+2)-1}{2^{\beta}}+1-\frac{1}{2^{\beta-1}}\right\rfloor \\
F_{r}^{\sigma}=\left\lfloor\frac{N+2^{\beta}+2-2}{2^{\sigma}}\right\rfloor=\left\lfloor\frac{N+2}{2^{\sigma}}+2^{\beta-\sigma}-\frac{1}{2^{\sigma-1}}\right\rfloor
\end{gathered}
$$

lead to By Lemma 2(P1)

$$
\left\lfloor\frac{N+2}{2^{\beta}}\right\rfloor+\left\lfloor 1-\frac{1}{2^{\beta-1}}\right\rfloor \leq F_{r}^{\beta} \leq\left\lfloor\frac{N+2}{2^{\beta}}\right\rfloor+\left\lfloor 1-\frac{1}{2^{\beta-1}}\right\rfloor+1 .
$$


Since $\beta>1$, it knows $\left\lfloor 1-\frac{1}{2^{\beta-1}}\right\rfloor=0$ and thus

$$
\left\lfloor\frac{N+2}{2^{\beta}}\right\rfloor \leq F_{r}^{\beta} \leq\left\lfloor\frac{N+2}{2^{\beta}}\right\rfloor+1
$$

which is

$$
F_{l}^{\beta} \leq F_{r}^{\beta} \leq F_{l}^{\beta}+1
$$

With this relationship, the values that $A_{l}^{\beta}$ and $A_{r}^{\beta}$ take are summarized in Table 4 and it can see that the results match to both Corollary 2 and this proposition.

Table 4. Values of $A_{l}^{\beta}$ and $A_{r}^{\beta}$

\begin{tabular}{lll}
\hline$F_{l}^{\beta}$ & $A_{l}^{\beta}$ & $A_{r}^{\beta}$ \\
\hline$F_{l}^{\beta}$ is odd & $A_{l}^{\beta}=F_{l}^{\beta}$ & $F_{r}^{\beta}=F_{l}^{\beta} \Rightarrow A_{r}^{\beta}=A_{l}^{\beta}$ \\
& $F_{r}^{\beta}=F_{l}^{\beta}+1 \Rightarrow A_{r}^{\beta}=A_{l}^{\beta}+2$ \\
$F_{l}^{\beta}$ is even & $F_{r}^{\beta}=F_{l}^{\beta} \Rightarrow A_{r}^{\beta}=A_{l}^{\beta}$ \\
& $A_{l}^{\beta}=F_{l}^{\beta}+1$ & $F_{r}^{\beta}=F_{l}^{\beta}+1 \Rightarrow A_{r}^{\beta}=A_{l}^{\beta}$ \\
\hline
\end{tabular}

Case 2. The odd numbers in the interval $I$ lie on different levels of T3. This time, $\operatorname{let} k=\left\lfloor\log _{2} N\right\rfloor-1$ and $j=\frac{N-2^{k+1}-1}{2}$; then $N=N_{(k, j)}$ lies at position $j$ on level $k$ of $T_{3}$. Since there are $2^{k}$ nodes on level $k$, it knows that $n_{\beta}=2^{\beta-1}$ nodes lie on 2 adjacent levels due to $1<\beta \leq k$. By Proposition 11 , node $N+2^{\beta}=N_{(k+1, \omega)}$ and $\omega<j$. The distribution of the nodes is illustrated as figure 3 .

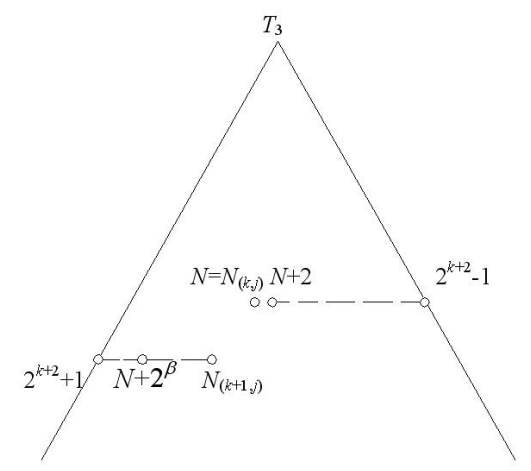

Figure 3. $2^{\beta-1}$ consecutive nodes lie on 2 adjacent levels of $T_{3}$

Assume there are $s$ nodes on level $k$ and $t$ nodes on level $k+1$; then $s+t=2^{\beta-1}$ and

$$
\begin{aligned}
& N=2^{k+2}-1-2 s=2^{k+2}-2 s-1 \\
& N+2=2^{k+2}-1-2(s-1)=2^{k+2}-2 s+1 \\
& N+2^{\beta}=2^{k+2}+1+2(t-1)=2^{k+2}+1+2(s-1)+2^{\beta}
\end{aligned}
$$

Now let $U=2^{k+2-\beta}-1, V=2^{k+2-\beta}+1$; then in $T_{U}$ and $T_{V}$ respectively it holds

$$
\begin{aligned}
& N_{\left(\beta, 2^{\beta}-1-(s-1)\right)}^{U}=2^{\beta}(U-1)+1+2\left(2^{\beta}-1-(s-1)\right) \\
& =2^{\beta}\left(2^{k+2-\beta}-1-1\right)+1+2\left(2^{\beta}-s\right) \\
& =2^{k+2}-2^{\beta+1}+1+2^{\beta+1}-2 s \\
& =2^{k+2}-2 s+1
\end{aligned}
$$

and

$$
\begin{aligned}
& N_{(\beta, t-1)}^{V}=2^{\beta}(V-1)+2(t-1)+1 \\
& =2^{k+2}+2\left(2^{\beta-1}-s-1\right)+1 \\
& =2^{k+2}+2^{\beta}-2 s-1=N+2^{\beta} .
\end{aligned}
$$


which shows $A_{N+2}^{\beta}=U$ and $A_{N+2^{\beta}}^{\beta}=V$. Since $V-U=2$, it knows the conclusion holds for Case 2 .

Proposition 13. Given a positive odd integer $N$ and a positive integer $\beta$ satisfying $1 \leq \beta<\left\lfloor\log _{2} N\right\rfloor$; let $X$ be an odd integer in odd interval $I=\left[N+2, N+2^{\beta}\right]$. Then

$$
A_{N+2}^{\alpha} \leq A_{X}^{\alpha} \leq A_{N+2}^{\alpha}+2^{\beta-\alpha}
$$

where $1 \leq \alpha<\beta$ is an integer.

Proof. Referring to Lemma 2(P13) and Corollary 2.

Proposition 14. Given a positive odd integer $N$ and a positive integer $\beta$ satisfying $1 \leq \beta<\left\lfloor\log _{2} N\right\rfloor$; let $X$ be an odd integer in odd interval $I=\left[N-2^{\beta}, N-2\right]$. Then

$$
A_{N-2}^{\beta}-2 \leq A_{X}^{\beta} \leq A_{N-2}^{\beta}
$$

and

$$
A_{N-2}^{\alpha}-2^{\beta-\alpha} \leq A_{X}^{\alpha} \leq A_{N-2}^{\alpha}
$$

where $1 \leq \alpha<\beta$ is an integer.

Proof. Referring to Proposition 13.

\section{Conclusion}

As stated in the introductory section, the aim of this paper is to show the newly-discovered important properties of the valuated binary tree for possible future studies. Why is it important? It is because the binary tree itself is a basic tool in data structure and referring to WANG X (2020b) it is naturally related with the blind search. Since the tree has many other undiscovered properties, future work on digging out new properties is necessary and worth to contribute. Hope more young to join the work.

\section{Acknowledgement}

The research is supported by Natural Science Foundation of Guangdong Province under Grant No. 2018A0303130082, the Open Project Program of the State Key Lab of CAD\&CG(Grant No. A2002) and Foshan Bureau of Science and Technology under project that constructs Guangdong Engineering Center of Intelligent Manufacturing System.

\section{References}

WANG, X. (2016a). Valuated Binary Tree: A New Approach in Study of Integers. International Journal of Scientific and Innovative Mathematical Research, 4(3), 63-67.

WANG, X. (2016b). Amusing Properties of Odd Numbers Derived From Valuated Binary Tree.IOSR Journal of Mathematics, 12(6), 17-24.

WANG, X. (2017a). Two More Symmetric Properties of Odd Numbers. IOSR Journal of Mathematics, 13(3-II), 37-40. https://doi.org/10.9790/5728-1303023740

WANG, X. (2017b). Genetic Traits of Odd Numbers With Applications in Factorization of Integers. Global Journal of Pure and Applied Mathematics, 13(2), 493-517.

WANG, X. (2018a). T3 Tree and Its Traits in Understanding Integers. Advances in Pure Mathematics, 8(5), 494-507. https://doi.org/10.4236/apm.2018.85028

WANG, X. (2018b). More on Square and Square Root of a Node on T3 Tree. International Journal of Mathematics and Statistics Study, 6(3), 1-7.

Wang, X., Guo, H., Alshaery, A., Alsulami, A., Robdera, M. A., Bildik, N., ... \& Yang, L. (2019). Some Divisibility Traits on Valuated Binary Trees. International Journal of Applied Physics and Mathematics, 9(1), 1-15. https://doi.org/10.17706/ijapm.2019.9.1.1-11

WANG, X. et al., (2019b). Traits of an RSA Modulus on T3 Tree. Journal of Mathematics Research, 10(6), 15-29. https://doi.org/10.5539/jmr.v10n6p15

WANG, X. et al., (2019c). Distribution of RSA Number's Divisor on T3 Tree.International Journal of Information and Electronics Engineering, 9(1), 23-29. https://doi.org/10.18178/IJIEE.2019.9.1.699

LI, J. (2018). A Parallel Probabilistic Approach to Factorize a Semiprime.American Journal of Computational Mathematics, 8(2), 175-183. https://doi.org/10.4236/ajcm.2018.82013 
WANG, X. (2018c). Influence of Divisor-ratio to Distribution of Semiprime's Divisor.Journal of Mathematics Research, 10(4), 54-61. https://doi.org/10.5539/jmr.v10n4p54

Dinesh, P. (2005). et al., Handbook of Data Structures and Applications. Chapman and Hall/CRC.

WANG, X. (2020a). Frequently-Used Properties of the Floor Function.International Journal of Applied Physics and Mathematics, 10(4), 135-142. https://doi.org/10.18178/ijiet.2020.10.7.1417

WANG, X. et al., (2020b). Traits of Interval Tree in Solving Blind Search Problems of Finding a Term in an Ordered Data Set.International Journal of Information and Education Technology, 10(7), 516-522.

\section{Copyrights}

Copyright for this article is retained by the author(s), with first publication rights granted to the journal.

This is an open-access article distributed under the terms and conditions of the Creative Commons Attribution license (http://creativecommons.org/licenses/by/4.0/). 